\title{
Routine colonoscopy may be needed for uncomplicated acute right colonic diverticulitis
}

\author{
Kil-yong Lee, Jaeim Lee* ${ }^{*}$, Youn Young Park and Seong Taek Oh
}

\begin{abstract}
Background: Routine colonoscopy is recommended to determine the coexistence of colon cancer after medical treatment for colon diverticulitis. However, in the case of uncomplicated diverticulitis diagnosed by computed tomography, the clinical relevance of routine follow-up colonoscopy has recently been debated. Yet, the role of follow-up colonoscopy for right colon diverticulitis, which tends to develop at a younger age than left colon diverticulitis, has not been specifically evaluated. Therefore, we aimed to evaluate the incidence of right colon cancer or colonic adenomatous polyps, detected by routine colonoscopy, after conservative management of acute uncomplicated right colon diverticulitis.
\end{abstract}

Methods: Patients with uncomplicated right colon diverticulitis (modified Hinchey stage la) diagnosed by computed tomography imaging, between 2011 and 2017, and who underwent follow-up colonoscopy surveillance after treatment were included. The primary outcome was the incidence of colon cancer, with the detection rate of adenoma being the secondary outcome. Information for analysis was retrieved retrospectively from patients' medical records.

Results: The study group included 330 consecutive patients, with a mean age of 41.9 years, and $51.9 \%$ being men. For the primary outcome, the rate of colon cancer on follow-up colonoscopy was $0.3 \%$ (1/330 cases). The rate of adenoma detection was 20.9\% (69/330 cases) and advanced adenoma (> 10 mm in diameter; or exhibiting a > 25\% villous component or severe dysplasia), including colon cancer, was observed in 9 patients (2.7\%).

Conclusions: In patients with acute uncomplicated right colonic diverticulitis, routine colonoscopy after conservative treatment may be necessary because although the colon cancer detection rate is low, it is possible to detect advanced colon adenoma.

Keywords: Diverticulitis, Acute diverticulitis, Colonic evaluation, Endoscopy, Colonoscopy, Colonic neoplasia

\section{Background}

The prevalence of diverticular disease has increased, both the in East and West, due to a lack of dietary fiber intake [1]. In contrast to complicated diverticulitis, which requires surgical treatment, uncomplicated acute diverticulitis can improve with conservative management,

\footnotetext{
*Correspondence: lji96@catholic.ac.kr

Division of Coloproctology, Department of Surgery, Uijeongbu St. Mary's

Hospital, College of Medicine, The Catholic University of Korea, 271,

Cheonbo-ro, Uijeongbu, Gyeonggi-do 11765, Korea
}

with colonoscopy recommended to identify accompanying disease, such as cancer [2]. Yet, a review article reported a low rate of cancer diagnosis during colonoscopy surveillance in patients treated for uncomplicated diverticulitis, with 1 case of cancer among the 67 cases included in the analysis [3]. However, these statistics are based solely on cases with left colon diverticulitis and only small number of patients (67 patients) were included. As such, the likelihood of cancer among patients treated with uncomplicated right colon diverticulitis is unknown. This knowledge, however, would 
be important as right-sided colon diverticulitis tends to occur at a younger age than left-sided colon diverticulitis, with a low stage Hinchey classification being common on computed tomography (CT) [4]. Generally, the American Cancer Society guideline recommends colonoscopy surveillance to begin at the age of 45 years [5]. Moreover, the onset of colon cancer at a younger age is more likely to occur in the left than right colon [6]. Considering these points, it is unclear how routine colonoscopy evaluation after right colon diverticulitis could be of clinical benefit with regard to diagnostic performance and economic burden.

Of the literature published on the effects of routine colonoscopy after the treatment of uncomplicated colon diverticulitis, few articles have exclusively included patients with right colon diverticulitis. Thus, the aim of our study was to evaluate the incidence of right colon cancer and colonic adenomatous polyps by routine colonoscopy, after conservative management of acute uncomplicated right colon diverticulitis.

\section{Methods}

We retrospectively reviewed the electronic medical records of patients diagnosed with acute right-sided colon diverticulitis, between January 2011 and December 2017, at our hospital.

Patients with acute uncomplicated diverticulitis confirmed by CT, were included. Acute uncomplicated diverticulitis was defined, in accordance with previous studies $[7,8]$, as the presence of colonic diverticular disease with localized colonic wall thickening and/or stranding of pericolonic fat. CT images with appearances of complicated diverticulitis (defined as the presence of pericolonic or abdominal abscess, localized or free extraluminal gas or contrast, obstruction or fistula formation) or the presence of an associated mass lesion were excluded. Followup colonoscopy was performed after improvement in the signs of inflammation corresponding to acute diverticulitis, with the consent of the patient. Exclusion criteria were as follow: complicated diverticulitis; accompanying left colon cancer; patients who underwent emergency surgery; and patients in whom follow-up colonoscopy was not performed or was not consented to. The reasons why a patient did not undergo colonoscopy was investigated based on a previous paper [9].

The following colonoscopy findings were documented: hyperplastic polyp, adenoma (including advanced adenoma), and carcinoma. Patients with polyps detected in the right colon were the focus of our analysis. Advanced adenoma was defined as either an adenoma of $10 \mathrm{~mm}$ or greater in diameter and/or more than $25 \%$ villous components and/or severe dysplasia [10].
The primary outcome was the detection rate of colon cancer, confirmed by pathological diagnosis, on followup colonoscopy. The secondary outcome was the detection rate of hyperplastic polyp and adenoma on follow-up colonoscopy.

\section{Statistical analysis}

Categorical variables were reported as a count (and associated percentage, \%), with continuous variables reported as the median and interquartile range (IQR). We analyzed the group with and without colonoscopy to know the characteristics of patients who have not performed the colonoscopy to be sure that the included patients are representative of the entire sample. To compare the group with and without colonoscopy, the chi-squared or Fisher exact test was used for categorical variables, as appropriate. As such, between-group comparisons of continuous variables were evaluated using the Mann-Whitney test. Additionally, the difference in the frequency of polyp detection was compared among patients under 45 years of age and over 45 years of age who underwent colonoscopy using the chi-squared or Fisher exact test.

All analyses were performed using SPSS (version 21, IBM, NY, USA).

\section{Results}

A total of 668 consecutive patients were diagnosed with acute right colon diverticulitis, by $\mathrm{CT}$, over the period of observation of the study. From these, the following patients were excluded from the analysis: 47 who had complicated diverticulitis; 1 with sigmoid colon cancer simultaneously diagnosed on CT imaging; and 1 who underwent emergency surgery because symptoms did not improve. Of the remaining 619 patients, follow-up colonoscopy was performed in 330 (Fig. 1). Follow-up colonoscopy was performed at a median of 32 days (IQR, 25-42 days) after recovery from acute diverticulitis.

Clinical characteristics of patients included in the analysis are reported in Table 1, with salient characteristics as follows: median age, 40 (IQR, 33-49) years; male, 59.7\%; and predominant involvement of the cecum (48.3\%) and ascending colon (49.9\%). With regard to clinical factors, only the length of hospital stay was significantly different between the two groups.

On follow-up colonoscopy, hyperplastic polyps were identified in 30 patients (9.1\%) and adenomas in 69 patients (20.9\%), with evidence of high-grade dysplasia in 2 of these cases. Villous adenoma was detected in 1 case. An advanced adenoma, including colon cancer, was observed in 9 patients (2.7\%), one of whom was diagnosed with ascending colon cancer. Polyps were found in the right colon in 46 of 87 patients with colon polyps (Table 2). Additionally, on biopsy, chronic inflammation 


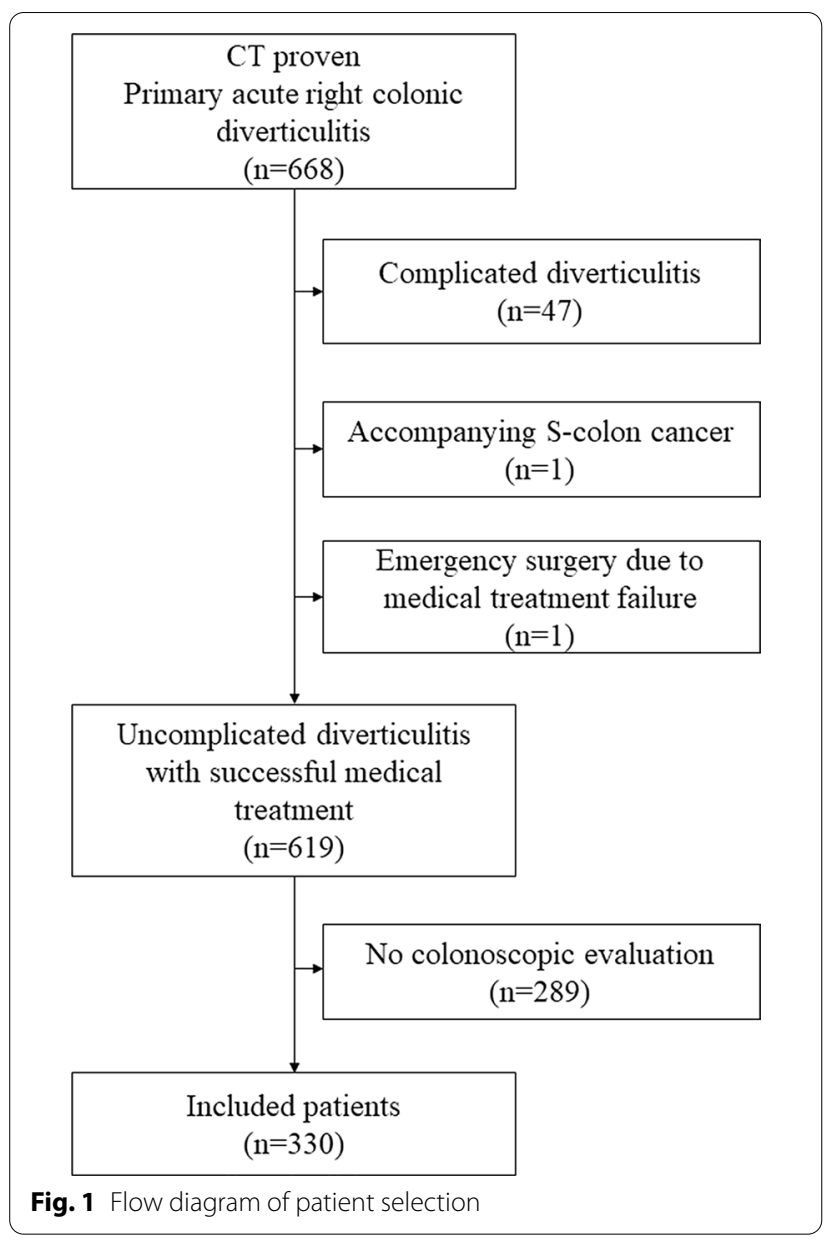

of the right colon was confirmed in 13 patients: erythematous mucosal change $(n=5)$ or ulceration $(n=8)$. Furthermore, right colonic diverticuli were found in 261 (79.1\%) of 330 patients who underwent colonoscopy. There was no incidence of inflammatory bowel disease combined with diverticulosis.

Ascending colon cancer was found in one male patient in his late $40 \mathrm{~s}$ at 6 weeks after he originally presented to our emergency department owing to a 2-day history of abdominal pain. CT examination, performed by an expert radiologist, revealed a segmented cecum and thickening of the wall of the ascending colon, with perilesional fat infiltration. He was admitted and treated with a 5-day course of intravenous antibiotics for a presumed diagnosis of uncomplicated right-sided acute diverticulitis. Follow-up colonoscopy was performed 6 weeks after imaging, with a diagnosis of ascending colon cancer confirmed.

In uncomplicated diverticulitis (330 patients) who underwent colonoscopy, recurrence occurred in 30 cases (9.1\%) for a median follow-up period of 680 days, and surgery was performed in a total of 5 cases (1.5\%) (in
4 cases for recurrent diverticulitis and in 1 case for the aforementioned cancer).

The reasons for lack of follow-up colonoscopy are summarized in Table 3, and main reasons were summarized as follows: 103 patients (35.6\%) scheduled colonoscopy, but not yet performed with unknown causes, 86 patients (29.8\%) were lost to follow-up, 22 patients $(7.8 \%)$ had a history of a recent prior colonoscopy; 15 (5.3\%) underwent follow-up colonoscopy at another hospital; and 10 (3.5\%) were followed up using colon barium study at the patient's request.

Since the frequency of polyp detection may vary according to age, we analyzed 330 patients who underwent colonoscopy into over 45 and under 45 years of age. As a result, the overall incidence of colonic polyp was $16.3 \%$ (34 patients) and $43.0 \%$ (52 patients) in patients under and over 45 years of age, respectively $(p<0.001)$. Specifically, adenoma was detected in $26(12.4 \%)$ and 43 (35.5\%) patients under and over 45 years of age, respectively $(p<0.001)$. Furthermore, hyperplastic polyp $(14.9 \%$ vs. $5.7 \%, p=0.005)$ and low grade dysplasia $(33.9 \%$ vs. $12.4 \%, p<0.001)$ were found more frequently in the age of 45 years and over. Most of all, the rate of advanced adenoma detection was statistically significantly higher in those aged 45 years or older than those under 45 years old. (5.8\% vs. $1.0 \%, p=0.009)$ (Table 4 ).

\section{Discussion}

The incidence rate of colon cancer detected by routine colonoscopy performed after conservative treatment of right colon acute uncomplicated diverticulitis was $0.3 \%$ (1/330 cases). The detection rate of adenoma in the whole colon was $20.9 \%$ and the frequency of advanced adenoma was $2.7 \%$. Specifically, the adenoma detection rate $(35.5 \%$ vs. $12.4 \%, p<0.001)$ and the rate of advanced adenoma (5.8\% vs. $1.0 \%, p=0.009$ ) were higher in those over 45 years old.

Clinically, the features of right colon diverticulitis are different from those of left colon diverticulitis. Specifically, compared to left colon diverticulitis, right colon diverticulitis tends to occur at a younger age, with a lower Hinchey stage, and a lower rate of recurrence (3.1\% vs. $17.9 \%$ for left colon diverticulitis) [11]. Based on these facts, and considering the high diagnostic yield of $\mathrm{CT}$ imaging and the development of accurate pathological finding for colon cancer, the utility of routine colonoscopy in patients after treatment of acute right colon diverticulitis, in the absence of complications, such as perforation, abscess formation, and/or obstruction, has been questioned [12]. It has also been questioned if the incidence of colorectal cancer at younger ages is indeed more common on the left than on the right colon [6]. 
Table 1 Clinical characteristics of patients with acute uncomplicated diverticulitis

\begin{tabular}{|c|c|c|c|}
\hline Variables & Colonoscopy $(n=330)$ & No colonoscopy $(n=289)$ & $p$ value \\
\hline Age (years) & $40(34-50)$ & $40(31-48)$ & $0.168^{d}$ \\
\hline Age group & & & $0.656^{\mathrm{e}}$ \\
\hline$<45$ (years) & $209(63.3 \%)$ & $188(65.1 \%)$ & \\
\hline$>45$ (years) & $121(36.7 \%)$ & $101(34.9 \%)$ & \\
\hline Sex & & & $0.778^{e}$ \\
\hline Male & $195(59.1 \%)$ & $174(60.2 \%)$ & \\
\hline Female & $135(40.9 \%)$ & $115(39.8 \%)$ & \\
\hline Height (cm) & $167.5(160.0-173.5)$ & $168.0(160.4-173)$ & $0.962^{d}$ \\
\hline $\mathrm{BMI}\left(\mathrm{kg} / \mathrm{m}^{2}\right)$ & $23.8(21.7-26.3)$ & $23.4(21.6-25.9)$ & $0.135^{d}$ \\
\hline Duration of hospital stay (days) & $4(4-5)$ & $4(3-5)$ & $0.010^{d}$ \\
\hline \multicolumn{4}{|l|}{ Social history } \\
\hline Smoking & $142(43 \%)^{\mathrm{a}}$ & $139(48.1 \%)$ & $0.303^{e}$ \\
\hline Alcohol & $137(42.7 \%)^{b}$ & $110(38.1 \%)^{c}$ & $0.248^{e}$ \\
\hline \multicolumn{4}{|l|}{ Past medical history } \\
\hline Hypertension & $50(15.2 \%)$ & $31(10.7 \%)$ & $0.103^{\mathrm{e}}$ \\
\hline Diabetes & $14(4.2 \%)$ & $12(4.2 \%)$ & $0.956^{\mathrm{e}}$ \\
\hline Location of the acute diverticulitis & & & $0.770^{e}$ \\
\hline Cecum & $154(46.7 \%)$ & $144(49.8 \%)$ & \\
\hline Ascending & $171(51.8 \%)$ & $139(48.1 \%)$ & \\
\hline Hepatic flexure & $3(0.9 \%)$ & $3(1.0 \%)$ & \\
\hline Transverse & $2(0.6 \%)$ & $3(1.0 \%)$ & \\
\hline White blood cell count $\left(10^{3} / \mu \mathrm{l}\right)$ & $11.1(9.2-12.9)$ & $11.5(9.7-14.0)$ & $0.082^{d}$ \\
\hline C-reactive protein (mg/dl) & $3.5(1.6-6.5)$ & $3.8(1.6-7.0)$ & $0.553^{d}$ \\
\hline
\end{tabular}

Continuous variables are reported as the median (and IQR)

Categorical variables are reported as a count (and percentage, \%)

$I Q R$ interquartile range, $B M I$ body mass index

a Missing data: $\mathrm{n}=1$

b Missing data: $\mathrm{n}=9$

c Missing data: $\mathrm{n}=13$

d Mann-Whitney test

e Chi-squared test

Table 2 Results of colonoscopy examination

\begin{tabular}{lc}
\hline Variables & Value \\
\hline Hyperplastic polyp & $30(9.1 \%)$ \\
Adenoma & $67(20.3 \%)$ \\
Low grade dysplasia & $2(0.6 \%)$ \\
High grade dysplasia & $1(0.3 \%)$ \\
Villous adenoma & $9(2.7 \%)$ \\
Advanced adenoma & $8(2.4 \%)$ \\
$>10$ mm in diameter & $1(0.3 \%)$ \\
$>25 \%$ villous components & $2(0.6 \%)$ \\
High grade dysplasia & $1(0.3 \%)$ \\
Adenocarcinoma & $46(52.9 \%)^{\mathrm{a}}$ \\
$\begin{array}{l}\text { Right-sided polyp } \\
\text { a Number of patients in whom polyps were detected in the right colon among }\end{array}$
\end{tabular}

Table 3 Reasons for patients to not undergo colonoscopy

\begin{tabular}{lc}
\hline Reasons & Number (\%) \\
\hline Prior colonoscopy $\leq 12$ months prior & $15(5.2 \%)$ \\
$12<$ Prior colonoscopy $\leq 24$ months & $3(1.0 \%)$ \\
$24<$ Prior colonoscopy $\leq 36$ months & $1(0.3 \%)$ \\
$36<$ Prior colonoscopy $\leq 60$ months & $2(0.7 \%)$ \\
$60<$ Prior colonoscopy $\leq 120$ months & $1(0.3 \%)$ \\
Patient declined & $12(4.2 \%)$ \\
Lost to follow-up & $86(29.8 \%)$ \\
Patient frailty & $2(0.7 \%)$ \\
No recommendation by treating team & $12(4.2 \%)$ \\
Not scheduled, reason unknown & $25(8.7 \%)$ \\
Colonoscopy scheduled, but have not had procedure & $103(35.6 \%)$ \\
with unknown cause & \\
Colonoscopy performed at another hospital & $15(5.2 \%)$ \\
Double-contrast barium enema only performed & $10(3.5 \%)$ \\
Death prior to colonoscopy & $1(0.4 \%)$
\end{tabular}


Table 4 Comparison of polyp detection during routine colonoscopy between under and over 45 years old

\begin{tabular}{llll}
\hline Variables & Under 45 (n= 209) & Over 45 (n= 121) & $\boldsymbol{p}$ value \\
\hline $\begin{array}{l}\text { Hyperplastic polyp } \\
\text { Adenoma }\end{array}$ & $12(5.7 \%)$ & $18(14.9 \%)$ & 0.005 \\
$\begin{array}{c}\text { Low grade dys- } \\
\text { plasia }\end{array}$ & $26(12.4 \%)$ & $41(33.9 \%)$ & $<0.001$ \\
$\begin{array}{c}\text { High grade dys- } \\
\text { plasia }\end{array}$ & $0(0 \%)$ & $2(1.7 \%)$ & 0.134 \\
$\begin{array}{l}\text { Adenocarcinoma } \\
\text { Advanced adenoma }\end{array}$ & $0(0 \%)$ & $1(0.8 \%)$ & 0.368 \\
\hline
\end{tabular}

Unlike left colonic diverticulitis, which is common in Westerners, right colonic diverticulitis is more common in the Asian population, and as mentioned above, the clinical manifestations are generally milder [4]. However, while there are numerous reports on the various management strategies for left colonic diverticulitis in the West, there are few studies on right colonic diverticulitis. Above all, reports on colonoscopic evaluation of right colonic diverticulitis remain insufficient. Therefore, our results regarding colonoscopic evaluation for uncomplicated right colonic diverticulitis could serve as a useful guideline for physicians treating Asian populations.

Several studies have been published on the effects of routine colonoscopy after conservative treatment of acute uncomplicated diverticulitis. Among 205 patients who underwent colonoscopy or CT colonoscopy for all colonic diverticulitis regardless of the part, Westwood et al. reported a detection rate of $9.3 \%$ for adenomas and $0.5 \%$ for colorectal cancer [13]. Additionally, Horesh et al. reported a rate of malignant findings of 1.6\% among 310 patients for all colonic diverticulitis who underwent colonoscopy. Of specific clinical relevance is the finding that there was no incidence of adenocarcinoma of the colon on follow-up colonoscopy after uncomplicated colon diverticulitis among patients younger than 50 years of age [14]. However, the majority of this evidence included only patients with left colon diverticulitis. In fact, to the best of our knowledge, only two previous studies addressed right colon diverticulitis $[15,16]$. In their study of 109 patients with right-sided colon diverticulitis, Hashimoto et al. did not identify any cases of colorectal cancer, with a rate of advanced adenoma of $6.4 \%$ (7/109 cases) and nonadvanced adenoma of $21.1 \%$ (23/109 cases) [16]. Chan et al. reported on 27 patients with right colon diverticulitis, with no incidence of colorectal cancer or advanced adenoma identified [15]. However, both of these studies included a small number of patients. By contrast, our study included 330 patients, a relatively large sample size. Similar to previous findings, adenoma and cancer detection rates were very low. Of significance was our finding that adenoma were identified only in the right colon.

This study was based on Korean. According to a previous population-based study about the prevalence of colorectal adenomas in asymptomatic Korean men and women published in 2014 [17], the prevalence of colorectal adenomas and advanced adenomas were 34.5\% and $3.1 \%$, respectively. Especially in subgroup analysis for under $<50$ years old, although the adenoma detection rate was $20.6-24.4 \%$, similar to that of this study (20.9\%), the rate of advanced adenoma detection was fairly higher in our study (2.7\%) than previous study (1.1-1.7\%). This suggests that patients with right uncomplicated colonic diverticulitis are more likely to have advanced colon adenoma, so routine colonoscopy should be performed.

The adenoma detection rate (ADR) has been associated with the interval risk of colorectal cancer [18]. The ADR can be used as a colonoscopy quality indicator, with an ADR of $<20 \%$ being associated with a 10 -fold increase in the interval cancer risk [19]. The ADR in our study, which included only patients with right colon diverticulitis, was $20.9 \%$. We consider this rate to be appropriate for our study as our primary outcome was the detection rate of colon cancer.

In the previous literature, it reported that complicated diverticulitis is more likely to be associated with colon cancer than uncomplicated diverticulitis [9]. Therefore, our study also included complicated diverticulitis and tried to compare it with uncomplicated diverticulitis. However, the number of patients with complicated diverticulitis $(n=47)$ was smaller than previous study $(\mathrm{n}=172)$. Of the 47 patients, 8 underwent emergency surgery and all patients were diagnosed with diverticulitis after surgery. In addition, only 21 out of 39 patients underwent routine colonoscopy, of which no colon cancer was found. (Additional file 1: Tables S1, S2). Therefore, complicated diverticulitis was excluded from our study.

\section{Limitation}

The limitations of our study need to be acknowledged. Foremost, this is a retrospective study, with no knowledge of the outcomes of colonoscopy surveillance for patients who did not undergo follow-up colonoscopy. We do note that patients who did not undergo followup colonoscopy tended to be younger than those who did undergo colonoscopy follow-up, although there was no statistical significance. Furthermore, the presence of colon cancer was ruled out in 10 patients who underwent barium enema and 15 who underwent colonoscopy 
at other hospitals; however, they were excluded because of insufficient data regarding adenoma detection. If these are included, a total of 355 patients (57.7\%) would have undergone surveillance for colon cancer. This rate of surveillance is comparable to previously reported rate in review articles [20,21]. Second, as our study is not a population based, our findings do not provide an estimate of colon cancer incidence in all patients with uncomplicated right colon diverticulitis. Third, since our study was conducted on Asian patients, it may not be applicable to non-Asian populations with right colonic diverticulitis. However, the strength of our study is the relatively large sample size which, in fact, is the largest study to date evaluating routine colonoscopy results among patients with right colon diverticulitis.

\section{Conclusions}

In patients with acute uncomplicated right colonic diverticulitis, routine colonoscopy after conservative treatment may be necessary because it is possible to detect advanced colon adenoma, even in those younger than 45 years old, who had an adenoma incidence rate of $12.4 \%$. Especially, for patients under 45 years of age with a family history, or patients over 45 years of age who have not undergone colonoscopy within the last 3 years, screening colonoscopy is strongly recommended, which is consistent with published guidelines [5]. It will be necessary to confirm the results of our study by collecting a larger number of patients through a multicenter or population based study.

\section{Supplementary Information}

The online version contains supplementary material available at https://doi. org/10.1186/s12876-021-01672-1.

Additional file 1: Clinical characteristics and adenoma detection rate between uncomplicated and complicated diverticulitis. Supplementary Table 1: Clinical characteristics between uncomplicated and complicated diverticulitis. Supplementary Table 2: Rate of detection of adenoma during routine colonoscopy between the uncomplicated and complicated diverticulitis groups.

\section{Abbreviations}

ADR: Adenoma detection rate; CT: Computed tomography; IQR: Interquartile range.

\section{Acknowledgements}

Not applicable.

\section{Authors' contributions}

KYL and JIL designed the study. KYL and YYP collected the data and performed the statistical analysis. KYL, JIL, YYP and STO interpreted the results of the analysis and prepared the manuscript. All authors contributed extensively to the work presented. All authors read and approved the final manuscript.

\section{Funding}

Not applicable.

\section{Availability of data and materials}

The datasets used and/or analysed during the current study available from the corresponding author on reasonable request.

\section{Ethics approval and consent to participate}

This study was approved by our hospital's institutional review board. Owing to the retrospective design of the study, the need for informed consent was waived. The study was conducted according to the principles of the Declaration of Helsinki.

\section{Consent for publication}

Not applicable.

\section{Competing interests}

The authors declare that they have no competing interest.

Received: 24 December 2019 Accepted: 17 February 2021

Published online: 27 February 2021

\section{References}

1. Painter NS, Burkitt DP. Diverticular disease of the colon: a deficiency disease of Western civilization. Br Med J. 1971;2(5759):450-4.

2. Strate LL, Morris AM. Epidemiology, pathophysiology, and treatment of diverticulitis. Gastroenterology. 2019;156(5):1282-98 e1281.

3. Strate LL, Peery AF, Neumann I. American Gastroenterological Association Institute Technical Review on the management of acute diverticulitis. Gastroenterology. 2015;149(7):1950-76 e1912.

4. Chung BH, Ha GW, Lee MR, Kim JH. Management of colonic diverticulitis tailored to location and severity: comparison of the right and the left colon. Ann Coloproctol. 2016;32(6):228-33.

5. Wolf AMD, Fontham ETH, Church TR, Flowers CR, Guerra CE, LaMonte SJ, Etzioni R, McKenna MT, Oeffinger KC, Shih YT, et al. Colorectal cancer screening for average-risk adults: 2018 guideline update from the American Cancer Society. CA Cancer J Clin. 2018;68(4):250-81.

6. Segev L, Kalady MF, Church JM. Left-sided dominance of early-onset colorectal cancers: a rationale for screening flexible sigmoidoscopy in the young. Dis Colon Rectum. 2018;61(8):897-902.

7. Hulnick DH, Megibow AJ, Balthazar EJ, Naidich DP, Bosniak MA. Computed tomography in the evaluation of diverticulitis. Radiology. 1984;152(2):491-5.

8. Doringer E. Computerized tomography of colonic diverticulitis. Crit Rev Diagn Imaging. 1992;33(5):421-35.

9. Suhardja TS, Norhadi S, Seah EZ, Rodgers-Wilson S. Is early colonoscopy after CT-diagnosed diverticulitis still necessary? Int J Colorectal Dis. 2017;32(4):485-9.

10. Force USPST. Screening for colorectal cancer: recommendation and rationale. Ann Intern Med. 2002;137(2):129-31.

11. Soh NYT, Teo NZ, Tan CJH, Rajaraman S, Tsang M, Ong CJM, Wijaya R. Perforated diverticulitis: is the right and left difference present here too? Int J Colorectal Dis. 2018;33(5):525-9.

12. Agarwal AK, Karanjawala BE, Maykel JA, Johnson EK, Steele SR. Routine colonic endoscopic evaluation following resolution of acute diverticulitis: is it necessary? World J Gastroenterol. 2014;20(35):12509-16.

13. Westwood DA, Eglinton TW, Frizelle FA. Routine colonoscopy following acute uncomplicated diverticulitis. Br J Surg. 2011;98(11):1630-4.

14. Horesh N, Saeed Y, Horesh H, Berger Y, Speter C, Pery R, Rosin D, Gutman M, Zmora O. Colonoscopy after the first episode of acute diverticulitis: challenging management paradigms. Tech Coloproctol. 2016;20(6):383-7

15. Chan DKH, Tan KK. There is no role for colonoscopy after diverticulitis among Asian patients less than 50 years of age. Gastrointest Tumors. 2017:3(3-4):136-40

16. Hashimoto R, Matsuda T, Chonan A. Colonoscopy after computed tomography-diagnosed acute right-sided diverticulitis may be unnecessary. J Clin Gastroenterol. 2016;50(7):611-2.

17. Yang MH, Rampal S, Sung J, Choi YH, Son HJ, Lee JH, Kim YH, Chang DK, Rhee PL, Rhee JC, et al. The prevalence of colorectal adenomas in asymptomatic Korean men and women. Cancer Epidemiol Biomarkers Prev. 2014;23(3):499-507. 
18. Corley DA, Jensen CD, Marks AR, Zhao WK, Lee JK, Doubeni CA, Zauber AG, de Boer J, Fireman BH, Schottinger JE, et al. Adenoma detection rate and risk of colorectal cancer and death. N Engl J Med. 2014;370(14):1298-306.

19. Kaminski MF, Regula J, Kraszewska E, Polkowski M, Wojciechowska U, Didkowska J, Zwierko M, Rupinski M, Nowacki MP, Butruk E. Quality indicators for colonoscopy and the risk of interval cancer. N Engl J Med. 2010;362(19):1795-803.

20. Sai VF, Velayos F, Neuhaus J, Westphalen AC. Colonoscopy after CT diagnosis of diverticulitis to exclude colon cancer: a systematic literature review. Radiology. 2012;263(2):383-90.
21. de Vries HS, Boerma D, Timmer R, van Ramshorst $B$, Dieleman LA, van Westreenen HL. Routine colonoscopy is not required in uncomplicated diverticulitis: a systematic review. Surg Endosc. 2014;28(7):2039-47.

\section{Publisher's note}

Springer Nature remains neutral with regard to jurisdictional claims in published maps and institutional affiliations.
Ready to submit your research? Choose BMC and benefit from:

- fast, convenient online submission

- thorough peer review by experienced researchers in your field

- rapid publication on acceptance

- support for research data, including large and complex data types

- gold Open Access which fosters wider collaboration and increased citations

- maximum visibility for your research: over $100 \mathrm{M}$ website views per year

At BMC, research is always in progress.

Learn more biomedcentral.com/submissions 